\title{
Modelo de co-creación en mypimes: estudio de caso en el sector lácteo de Bogotá, Colombia*
}

\author{
Olga Alexandra Rodríguez-Chala McS* \\ Giovanny Mauricio Tarazona-Bermudez Ph.D.*** \\ Lillyana María Giraldo M.Ph.D. ${ }^{* * *}$
}

Recibido: 24/04/2018 • Aceptado:15/07/2020
https://doi.org/10.22395/rium.v20n38a14

\begin{abstract}
Resumen
Las micro y pequeñas empresas en Colombia requieren fortalecer sus ventajas competitivas, sostenibles y sustentables para responder, adaptarse y sostenerse en el mercado; deben concentrar sus esfuerzos en la gestión del conocimiento, la innovación las TIC, el mejoramiento continuo, y la co-creación. Este documento presenta un modelo de co-creación basado en la gestión del conocimiento que permita innovar en el sector lácteo como forma de adaptación y supervivencia, así como de diferenciación y competitividad como herramientas para la toma de decisiones. La metodología adoptada para resolver el problema planteado y comprobar la hipótesis de investigación combina situaciones y actitudes predominantes que proponen soluciones y alternativas de evaluación para el desarrollo de estrategias que permitan generar ventajas competitivas y mejorar continuamente los procesos de las mipymes (micro, pequeñas y medianas empresas) del sector lácteo y que como consecuencia disminuyan las dificultades socioeconómicas que aquejan a estas mipymes.
\end{abstract}

* Artículo derivado de la investigación CocreaciónenMipymes del sector lácteo en Bogotá: modelo de procesos de aprendizaje (Co-Creation in MSMEsIn The Dairy Production Sector in Bogota: Learning Processes Model) hecho como trabajo de fin de Máster en Ingeniería Industrial en la Universidad Distrital Francisco José de Caldas, entre junio 2015y agosto de 2016. Investigación financiada con recursos del centro de Desarrollo Científico e Investigaciones.

** Ingeniera industrial, Máster en Ingeniería Industrial y experta en gestión de calidad, gestión del riesgo e implementación de sistemas integrados de gestión en la Industria Militar Colombiana y su desarrollo continuo en procesos de automatización. Investigadora en el grupo Gicoecol (Grupo de Investigación en Comercio Electrónico Colombiano). Correo electrónico: oarodriguezc@correo.udistrital.edu.co. Orcid: https://orcid. org/0000-0003-3996-8396

*** Ingeniero Industrial, Máster en Diseño y Desarrollo de Proyectos Tecnológicos, Ph.D. en Sistemas Computacionales, Especialista en Ingeniería de software. Investigador en el grupo Gicoecol. Profesor Asociado en la Facultad de Ingeniería de la Universidad Distrital Francisco José de Caldas. Correo electrónico: gtarazona@ udistrital.edu.co. Orcid: https://orcid.org/0000-0001-5012-1466

**** Ingeniera de sistemas, Ph.D. Tecnologías de la Información y la Comunicación, Profesora en la Facultad de Ingeniería de la Universidad de Medellín, Colombia. Correo electrónico: 1mgiraldo@udem.edu.co. Orcid: https:// orcid.org/0000-0003-3496-6455 
Para el análisis de los modelos de competitividad de las mipymes del sector lácteo en Bogotá, se utilizó la metodología adoptada por grupo de investigación "Comercio electrónico en Colombia” (Gicoecol), se contextualiza la problemática de competitividad de las mipymes del sector lácteo, así como aspectos de procesos de aprendizaje, creación y/o personalización de productos y/o servicios ofrecidos, y aplicaciones de este tipo de modelos en otros sectores o en grandes empresas. Con base en la información recolectada a través de la etapa de análisis, se estructura un modelo basado en la gestión del conocimiento y co-creación de valor con el fin de ser una herramienta base para mejorar la competitividad, de que se generen estrategias en los niveles interno y externo y se estandaricen y formalicen sus procesos e incrementen su rentabilidad, se identificaron variables fundamentales para la construcción del modelo; por último, se realizó la validación de la propuesta mediante la medición de la aceptación del modelo propuesto.

Palabras Clave: gestión de conocimiento, innovación, co-creación, lácteos, competitividad, mipymes, sostenibilidad, mejoramiento continuo, participación, adaptabilidad.

\title{
Msmes Co-Creation Model: Case Study in the Dairy Sector of Bogota, Colombia
}

\begin{abstract}
Micro and small companies in Colombia need to strengthen their competitive, sustainable and sustainable advantages to respond, adapt and sustain themselves in the market; they must concentrate their efforts on knowledge management, ICT innovation, continuous improvement, and co-Creation. This document presents a co-creation model based on knowledge management that allows innovating in the dairy sector, as a form of adaptation and survival, of differentiation and competitiveness as well as a tool for decision-making. The methodology adopted to solve the problem posed and to check the research hypothesis combines situations and predominant attitudes that propose solutions and evaluation alternatives for the development of strategies that allow generating competitive advantages and continuously improving the processes of Msmes in the dairy sector, and that, as a consequence, decrease the socioeconomic difficulties that afflict these Msmes.
\end{abstract}

For the analysis of the competitiveness models of Msmes in the dairy sector in Bogota, the methodology adopted by the research group "Electronic Commerce in Colombia" (Gicoecol), was used to contextualize the competitiveness problem of Msmes in the dairy sector, as well as aspects of learning processes, creation and/or personalization of products and/or services offered, and applications of this type of model in other sectors or in large companies. Based on the information collected through the analysis stage, the study structured a model based on knowledge management and value co-creation, in order to be a base tool to improve competitiveness, generate strategies at an internal and external level, standardize and formalize their processes and increase their profitability; fundamental variables were identified for the construction of the model; Finally, the proposal was validated by measuring the acceptance of the proposed model.

Key Words: knowledge management; innovation; co-creation; dairy; competitiveness; MSME; sustainability; continuous improvement; participation; adaptability. 


\section{INTRODUCCIÓN}

Las mipymes (Micro y pequeñas empresas) no disponen de mecanismos de generación de conocimiento interno, están obligadas a adquirirlo externamente a través de diferentes mecanismos, lo que les obliga a trabajar conjuntamente con clientes, proveedores, universidades y centros de investigación [1]. En concordancia, las mipymes deben utilizar la co-creación como estrategia de aprendizaje, para que puedan hacer frente a las crecientes demandas del mercado, de tal manera que los clientes se mantengan en contacto con sus proveedores de servicios o productos e interactúen constantemente para obtener beneficios [2].

Dentro de los problemas identificados en cuanto al crecimiento y mejoramiento continuo se han podido identificar los siguientes:

- La falta de transparencia acerca del capital intelectual y de los activos que poseen o quieren desarrollar dificulta la obtención de recursos en las empresas [1-9]; por tanto, el conjunto de barreras para la inversión en investigación, desarrollo e innovación con el que se enfrentan las mipymes pueden ser agrupados en cuatro procesos [3]: escasez de recursos financieros, de conocimiento, de capital humano y de competencias gerenciales.

- La dificultad para analizar e identificar las competencias para adaptarse a las necesidades del mercado o ser competitivos $[1,6,7,10]$.

La competitividad como condición necesaria no debe ser un objetivo de corto o mediano plazo, sino la búsqueda de una condición sostenible, caracterizada por su permanencia en el mercado [9], [11-15], con la coexistencia de varios actores entre los que se destaca el sector productivo, el gobierno y los clientes.

Las principales problemáticas que las mipymes enfrentan para su desarrollo son la identificación de necesidades de mercado, la formalización y absorción de nuevas tecnologías, la formación de los colaboradores, las limitaciones competitivas que imponen las escalas de producción, la falta de asociatividad empresarial y la carencia de directivos con capacidad de pensamiento estratégico [2, 16, 17]. Cada vez es más evidente la necesidad de integrar a las empresas en redes para solucionar los problemas de competitividad, en este sentido, los clientes deben generar ideas con el proveedor, para definir el producto, desarrollar nuevos conceptos y procesos, generar productos piloto y renegociar las condiciones que consideren necesarias [18].

Las mipymes del sector lácteo pueden adaptarse mejor a su entorno y anticiparse a los competidores para mejorar sus resultados si tienen en cuenta la innovación como una práctica integrada en un nivel global [19,9], ya que las empresas no aportan valor, 
sino que dan propuestas de valor y es el cliente el que determina el valor y co-crea con la firma [13]; la gran problemática que enfrentan los empresarios de las mipymes es la falta de conocimiento sobre la investigación, desarrollo e innovación integrados para la identificación de necesidades y la creación de nuevos productos o servicios [20-22].

\section{METODOLOGÍA DE LA INVESTIGACIÓN}

La metodología adoptada para resolver el problema planteado y comprobar la hipótesis de investigación es una metodología mixta, ya que combina la metodología descriptiva que consiste en llegar a conocer las situaciones y actitudes predominantes a través de la descripción exacta de las actividades, procesos y personas; y la metodología proyectiva, que propone soluciones y alternativas de evaluación para el desarrollo de estrategias que permitan generar ventajas competitivas y mejorar continuamente los procesos de las mipymes del sector lácteo en Bogotá y que como consecuencia disminuyan las dificultades socioeconómicas que aquejan a estas mipymes.

Esta propuesta metodológica se caracteriza por articular los diferentes componentes del proyecto con el propósito principal de responder al problema de investigación a partir del cumplimiento de los objetivos desde una perspectiva teórica.

Para lograr los objetivos de la investigación, se utilizaron diversas herramientas metodológicas. Para el análisis de los modelos de competitividad de las mipymes del sector lácteo en Bogotá se utilizó la metodología adoptada por el grupo de investigación "Comercio Electrónico en Colombia" (Gicoecol), y así proponer un modelo que permita incrementar la competitividad de las mipymes del sector lácteo. Se utilizó una metodología de acción participativa en donde las empresas en estudio contribuyeron a la construcción del modelo que permitió generar ventajas competitivas y mejorar continuamente.

En la validación del modelo propuesto se realizó un panel de expertos y se utilizaron redes sociales para interactuar con los miembros del sector lácteo en Bogotá. La metodología exige unos pasos iterativos que combinan los distintos elementos conceptuales para generar como resultado un modelo que contribuya a la generación de ventajas competitivas y mejoramiento continuo, y del que, además, se extraen eventualmente conclusiones cualitativas. 

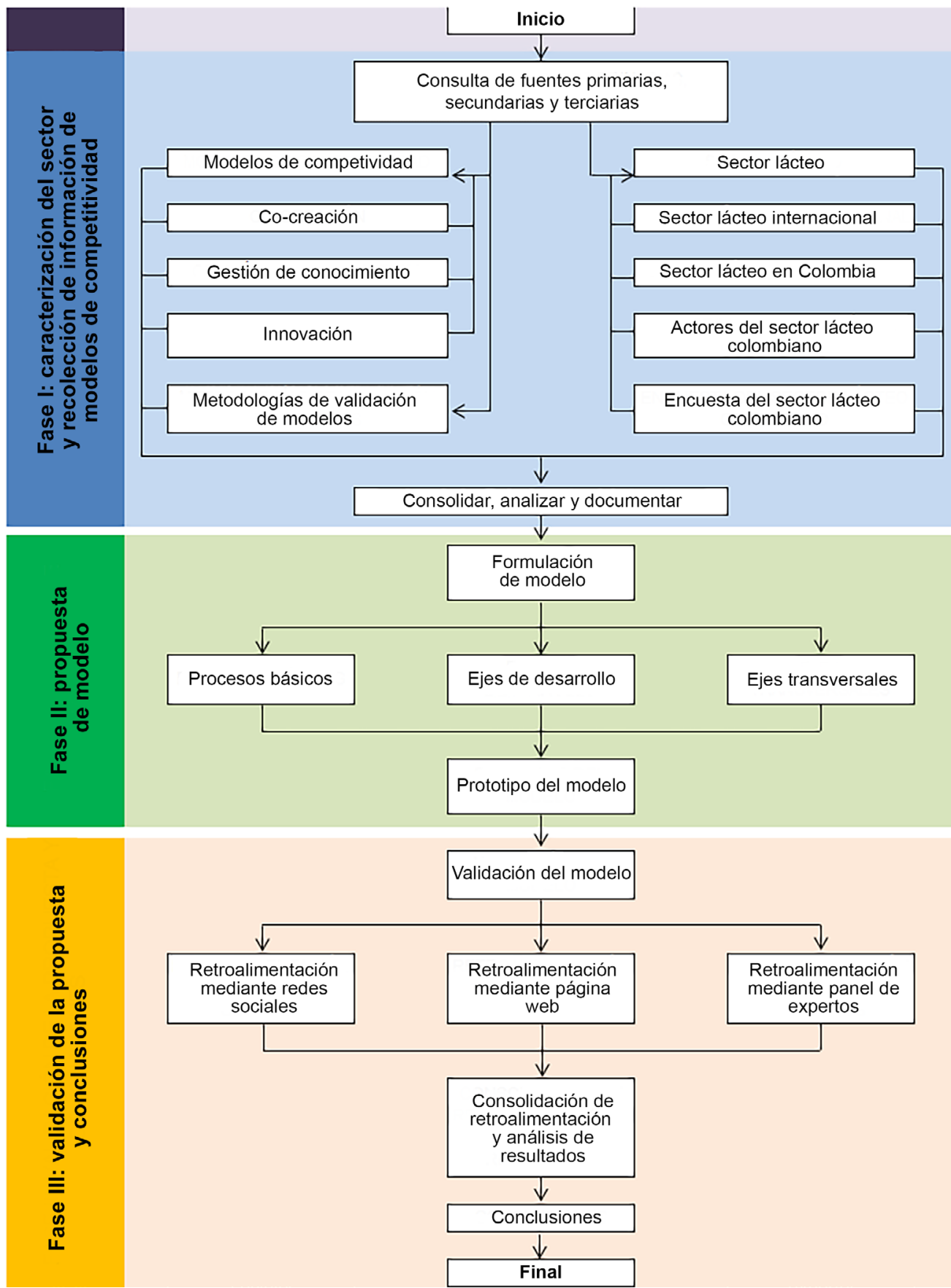

Figura 1. Desarrollo de la metodología

Fuente: elaboración propia. 
La metodología de acción participativa con las empresas en estudio permitió la construcción del modelo sustentado en la mejora continua. En la validación del modelo se realizó un panel de expertos y se utilizaron redes sociales para interactuar con los miembros del sector lácteo. Este se desarrolló en tres fases:

\section{FASE I: Caracterización del sector lácteo y recolección de información de modelos de competitividad}

Esta fase contextualiza la problemática de competitividad de las mipymes del sector lácteo, así como aspectos de procesos de aprendizaje, creación y/o personalización de productos y/o servicios ofrecidos y aplicaciones de este tipo de modelos en otros sectores o en grandes empresas.

\section{FASE II: Construcción y propuesta de modelo}

Con base en la información recolectada a través de la etapa de análisis se estructuró un modelo basado en la gestión del conocimiento y co-creación de valor y con el fin de ser una herramienta base para que las empresas incrementen su competitividad, generen estrategias a nivel interno y externo, estandaricen y formalicen sus procesos e incrementen su rentabilidad, se identificaron variables fundamentales para la construcción del modelo.

\section{FASE III: Validación de la propuesta mediante la medición de la aceptación del modelo propuesto}

Este proceso se enfocó en realizar una contrastación conceptual del modelo planteado, adaptando la metodología de validación por niveles planteada, que establece diferentes niveles y métodos de comprobación con el objetivo de asegurar una validación exhaustiva soportando diferentes aspectos y componentes del modelo propuesto.

Para esta fase se generó una página en la red social Facebook y una página web en una plataforma gratuita. Adicionalmente, se realizó un panel de expertos con el propósito de conocer la opinión de los actores del sector lácteo acerca del modelo propuesto.

\section{ASPECTOS TEÓRICOS DE LA INNOVACIÓN}

\subsection{Innovación}

La innovación de productos es la introducción de un nuevo producto, o un cambio cualitativo importante en un producto existente. La innovación de procesos es la introducción de un nuevo proceso para la fabricación o entrega de bienes y servicios. La innovación es el producto o proceso que debe ser introducido en el mercado para 
que los consumidores u otras empresas se puedan beneficiar. Esto distingue a una innovación de una invención o descubrimiento [23-25].

La innovación se concreta en el mercado con la colocación de nuevos productos y servicios de procesos derivados que aplican pragmáticamente los conocimientos existentes e implementando nuevos conocimientos Así, la innovación se produce en el núcleo de un proceso complejo, antepuesto de las invenciones y tiene éxito por la amplia adopción de la nueva innovación [26].

Existen modelos de innovación. Herramientas y software son mecanismos diseñados para apoyar las actividades de innovación y colaboración. Sin embargo, en muchos casos, los modelos de innovación tienden a limitarse o su aplicación no es rentable para las mypimes [27].

\subsection{Gestión del Conocimiento}

La gestión del conocimiento del cliente es un recurso estratégico para las empresas que permite mejorar la innovación, facilitar la detección de nuevas oportunidades de mercado y apoyar la gestión de relación con el cliente [28-29].

La creación de redes y la integración de la empresa, así como los sistemas de información empresarial orientados a la interoperabilidad, aumentan la productividad y eficiencia como consecuencia de la promesa de un intercambio de información más automatizado en los escenarios de las empresas en red. La integración entre los aspectos tecnológicos y sociales, vinculando las actividades que se asocian comúnmente con dicha gestión a otros procesos de organización de la naturaleza social, por ejemplo, aprendizaje, enseñanza, entrenamiento, etc., permite la alineación de la iniciativa de gestión del conocimiento con la estrategia y cultura organizacional [30].

Las tecnologías de la información y comunicación (TIC) juegan un papel en este proceso [31]. Por lo tanto, a medida que se establece una propuesta de modelo teórico, las TIC permiten transformar el conocimiento tácito en explícito. Este conocimiento se integra después en la cultura organizacional y requiere factores motivacionales para apropiarlo [32, 33].

El proceso de co-aprendizaje se fomenta debido al conocimiento transmitido y se codifica a través del diálogo entre los empleados, por lo tanto, se convierte en conocimiento explícito que puede ser compartido. La gestión del conocimiento puede ser analizada para entender el papel del ciclo de vida de gestión del conocimiento en cuatro fases: socialización, exteriorización, combinación e internalización [30], que permitan divulgar el conocimiento en las diversas área organizacionales. 


\subsection{Co-creación}

Desde la teoría de prosumidores propuesta por AlvinToffler, se inició una serie de hipótesis por parte de diversos investigadores como John A. Czepiel (profesor de marketing de la Escuela de Negocios de Nueva York), quien sugirió la participación del cliente como elemento principal hacia la satisfacción del cliente $[34,35]$.

Por otra parte, Scott Kelley, James Donnelly y Steven J. Skinner sugirieron que la participación del cliente generaría grandes beneficios para los productores, no solo en términos de calidad sino en las respuestas emocionales por parte del cliente y en aumento de la productividad debido al incremento de la eficiencia de los colaboradores [36-38].

De acuerdo con lo anterior, desde la perspectiva actual se generaron avances que permiten a las mipymes adaptarse mejor a su entorno y anticiparse a los competidores para mejorar sus resultados si tienen en cuenta la innovación como una práctica integrada en un nivel global [9], ya que las empresas no aportan valor, sino que dan propuestas de valor y es el cliente el que determina el valor y lo concreta con nuevas adquisiciones [13].

En el proceso de conversión del cliente en un co-creador del contenido de sus experiencias como base de la innovación, él es el actor principal de la creación participativa. Este modelo fue propuesto centrado en la experiencia de usuario como base fundamental de las innovaciones en un nivel global $[2,39]$.

Sobre el año 2000, Prahalad y Ramaswamy observaron la variabilidad de los roles entre empresa y consumidor, concluyendo que el cliente toma un papel activo para las empresas. Sugirieron aprovechar las competencias de los consumidores a lo largo de los siguientes ejes: entablar diálogo con los clientes, movilizar a las comunidades, gestionar la diversidad de clientes, co-crear y personalizar experiencias con los clientes $[38,40]$.

La participación activa de las partes interesadas en el ajuste, personalización o invención de soluciones para las empresas es el foco de la opinión de la co-creación. En este enfoque, la perspectiva de un solo inventor se sustituye por un proceso de flujo de conocimiento (entrada y salida) entre los grupos de interés como socios, productos, servicios y las experiencias se desarrollan en forma conjunta por las empresas y sus clientes [41].

Las tecnologías han creado nuevos modos de producción y de innovación que permiten y fomentan mayores grados de participación y colaboración [42, 22], [43-46], que las empresas deben utilizar a su favor para conocer a sus clientes y atacar con éxito. Al hacerlo se obtendrá una ventaja competitiva. 


\section{PROBLEMÁTICA DE LAS EMPRESAS DEL SECTOR LÁCTEO EN COLOMBIA}

La problemática del sector es de carácter estructural, mediada siempre por factores de rezago tecnológico, de estacionalidad climática y de un conflicto de precios, desarticulación entre los productores y la industria procesadora, el contrabando, la entrada masiva de derivados lácteos, la entrada en vigor de tratados de libre comercio, dependencia de insumos extranjeros, la falta de asociatividad y la poca capacidad de inversión en cadena de frío y plantas pulverizadoras [47]. Lo anterior puede resumirse de la siguiente manera:

- Existen vacíos en la regulación sobre derivados lácteos, es decir, no hay reglas claras respecto a la diferenciación que estos productos deben tener en su empaque y rotulado.

- La comercialización de la leche cruda es la principal fuente de informalidad. Sus causas son las dificultades en la infraestructura social, los problemas de orden público, desempleo y subempleo, inversión y costos altos para cumplir con normatividad vigente [48].

La informalidad varía de acuerdo con la región. En el caso de los departamentos predominantemente rurales y poco urbanizados existen altos índices de informalidad [49].

Entre las consecuencias de la informalidad se encuentran, principalmente, una distorsión en el precio pagado al productor, ya que los agentes comerciales ya no reportan el precio pagado a las entidades regulatorias MADR (Ministerio de Agricultura y Desarrollo Rural), lo que es obligación según la Resolución 17 del 2012. Además, el bajo consumo de leche en estratos bajos (1, 2 y 3 ), asociado con alto consumo de leche cruda, trae como consecuencia bajos niveles de nutrición y riesgo para la salud humana.

- El incremento constante de los costos de producción ganadera y el precio de los insumos ganaderos es el mayor de América. A los comercializadores de insumos agropecuarios se les disminuyen aranceles pero no se garantiza reducción de precios al ganadero.

- No se cuenta con un adecuado sistema de infraestructura de frío que permita a los campesinos permanecer un mayor tiempo con el producto, por lo que deben salir a ofertarlo casi de manera inmediata.

- Falta asociación entre los eslabones de la cadena, lo que genera un crecimiento independiente de cada uno. 
- Los laboratorios de análisis que determinan la calidad composicional de la leche son en su mayoría propiedad del mismo agente comprador, por lo que éste termina siendo juez y parte en el análisis de la calidad del producto, afectando de forma directa el principal determinante en la formación del precio a pagar.

- Existen diversos TLC (tratados de libro comercio) suscritos que facilitan la importación de leche y productos lácteos, pero no existe acceso real a esos mercados debido a las barreras sanitarias.

- Oligopolio en el mercado de la leche. Hay una gran cantidad de oferentes (ganaderos) y pocos compradores (industriales). Cinco compañías acopian cerca del $60 \%$ de la producción formal.

- Contrabando. El ingreso de leche en polvo de contrabando se presenta principalmente en la frontera con Venezuela. Del total de toneladas aprehendidas el $74 \%$ ingresa por las ciudades de Bucaramanga y Cúcuta, seguido por las ciudades de Santa Marta, Riohacha y Valledupar.

La actual capacidad instalada de la industria nacional se encuentra cerca de sus niveles máximos de utilización, por lo cual se estima que solo podrían aumentar sus volúmenes de compra y procesamiento de leche fresca en un $10 \%$.

Existe además el compromiso del Estado por asegurar que la totalidad de la leche fresca que se produce en Colombia sea acopiada, procesada y comercializada por industrias formalmente constituidas (Decreto 616 de 2006 y Decreto 3411 de septiembre de 2008), a través de la expansión de la capacidad transformadora del país mediante la puesta en funcionamiento de nuevas plantas de procesamiento de leche.

En este sentido, el proceso de formalización de la cadena láctea en Colombia representa una valiosa oportunidad de para el sector [49].

\section{PROPUESTA DE COCREACIÓN EN MIPYMES DEL SECTOR LÁCTEO: MODELO DE PROCESOS DE APRENDIZAJE}

Un modelo de co-creación en mipymes del sector lácteo basado en procesos de aprendizaje, permite proyectar una estrategia basada en la innovación. El modelo se basa en el fortalecimiento de los procesos de las mipymes del sector lácteo a través de ocho ejes fundamentales que sustentan esta investigación, para permitir un resultado final que resulte integral y que asegure una red empresarial sostenible en el tiempo. 
Además, el modelo está basado en cuatro bloques de construcción a saber: diálogo, acceso, riesgo y transparencia, entre todos los procesos y actores de la cadena del sector lácteo.

1. Diálogo: en múltiples puntos y con diferentes parejas dentro de la red de valor, fomenta el intercambio de conocimientos y una comprensión mutua entre las empresas, proveedores y clientes. Esto proporciona una oportunidad para que los clientes interpongan su visión de valor en el proceso.

2. Acceso: proporcionar acceso a todos los actores de la cadena láctea a los recursos, la información, las herramientas y procesos activos en varios puntos a través de la red de valor. Proporciona a las empresas nuevas oportunidades de negocio.

3. Riesgo: teniendo en cuenta que los clientes se convierten en co-creadores de valor, se vuelven más vulnerables al riesgo y comenzarán a exigir más información acerca de los riesgos potenciales asociados con el diseño, fabricación, suministro y consumo de bienes y servicios particulares.

4. Transparencia: crear la confianza entre los individuos. La transparencia es cada vez más un componente de las estrategias de diferenciación.

\subsection{Procesos básicos}

Las empresas implementan los siguientes procesos básicos y a su vez cada uno de los procesos genera datos e información, creando una cultura de conocimiento:

- Dirección: gestión de recursos y planeación estratégica para que la empresa alcance los objetivos de co-creación.

- Calidad e inocuidad: se consolida información de quejas y reclamaciones de los clientes en interacción con los demás procesos, se detectan factores propios de la materia prima, procesamiento, empaque, transporte y entrega que afectan la calidad del producto final o de servicio en las entregas a clientes.

- Investigación, desarrollo e innovación: Ejerce acción recíproca con los demás procesos para identificar necesidades de los proveedores, proceso, clientes y clientes potenciales.

- Gestión del conocimiento: consolida datos de todos los procesos y procedimientos de la empresa y propone metodologías para documentar el cómo hacer y lecciones aprendidas. 


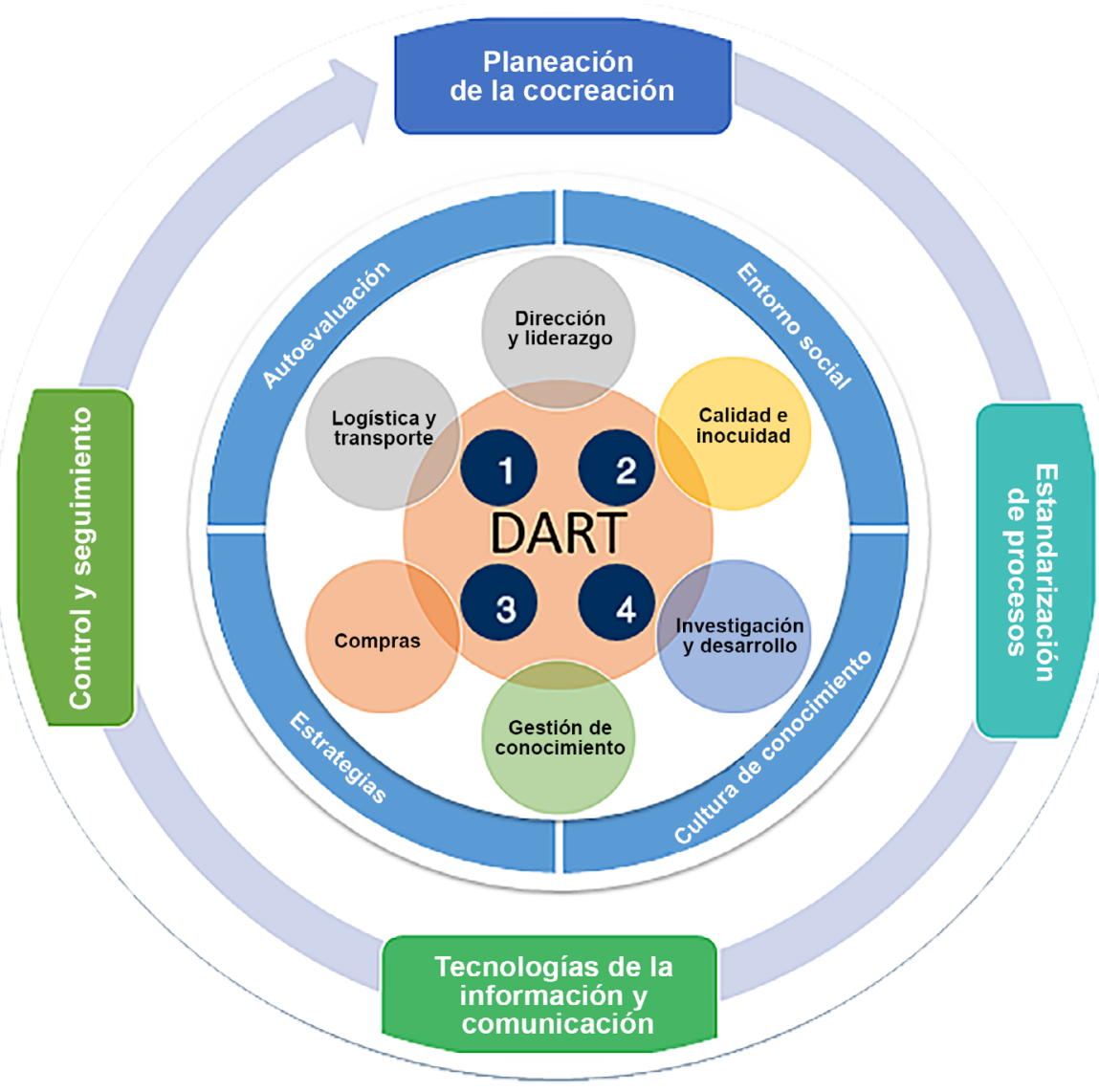

Figura 2. Modelo propuesto.

Fuente: Elaboración propia.

- Mercadeo: consolida datos de los clientes, ventas y clientes potenciales. Interactúa con los demás procesos y los procesos de los proveedores para calcular los precios de los productos, conocer los beneficios y diferenciadores con respecto a las demás empresas.

- Logística: consolida datos de precios de productos, condiciones de entrega de los proveedores, las rutas que se toman para la entrega de productos a la empresa y rutas para la entrega de productos a clientes.

\subsection{Ejes de desarrollo}

Los ejes de desarrollo son la cultura de conocimiento, el entorno social, la autoevaluación y las estrategias, los cuales buscan brindar herramientas que sustenten su aplicación para el fortalecimiento de las mipymes del sector lácteo. 
Eje de desarrollo social: las empresas deben trabajar para conocer las necesidades y las expectativas de sus clientes y ofrecerles las mejores soluciones, defendiendo el buen funcionamiento del mercado en libre competencia, esforzándose continuamente por cuidar e incrementar su satisfacción, reforzar su vinculación a la empresa y a través de tomar acción de acuerdo con la percepción.

Es necesario apoyar a los proveedores en la adopción de prácticas responsables para la gestión de la cadena de suministro, las prácticas laborales en la salud laboral y seguridad industrial, el medio ambiente, la calidad y seguridad de los productos y servicios comercializados, así como desarrollar prácticas responsables en la cadena de valor.

Eje de desarrollo del conocimiento: tiene como objetivo establecer fundamentos que permitan estructurar la seguridad e iniciativa de participación desde diversos puntos de vista con respecto a la actividad de los integrantes del sector, permitiendo la interacción entre los actores del sistema a través de TIC que logren un desarrollo competitivo que gire en torno al suministro de productos lácteos de calidad. La cultura del conocimiento se debe adquirir a medida que se van recolectando datos y se van procesando (crear información).

Eje de desarrollo de autoevaluación: permite conocer la opinión de los empleados acerca del funcionamiento interno, además, los trabajadores pueden expresar su opinión, sus problemas e ideas para la mejora. Es importante comunicar al personal los resultados de la autoevaluación y las acciones que se tomarán para corregir los problemas e implantar mejoras. Las autoevaluaciones se pueden hacer de manera periódica, continua (a través de resultados de indicadores) o puntuales (en la implementación de procesos o actividades para conocer la opinión de los empleados para buscar posibles mejoras).

Eje de desarrollo de estrategias: busca determinar las actividades a seguir para alcanzar los objetivos o metas de la empresa, de acuerdo con un análisis principal que determina nuestras debilidades, oportunidades, fortalezas y amenazas para poder lograr un objetivo común, basado en factores como productos o servicios a ofrecer, necesidad a satisfacer, segmentación de clientes, tecnología que se debe utilizar o desarrollar, marketing y zona geográfica.

\subsection{Ejes transversales}

Los ejes planeación de la co-creación, estandarización de procesos, TIC y control y seguimiento son los transversales.

Planeación de la co-creación: la co-creación se puede considerar el cimento sobre el cual se sustentan los demás ejes; la planeación de la co-creación genera pronósticos competitivos para las organizaciones, anticipa los riesgos, beneficios, oportunidades y 
falencias, para con base a ellos fijar una técnica que permita actuar en función de la innovación participativa prevista y así aprovechar al máximo las oportunidades detectadas para mitigar los riesgos.

Estandarización de los procesos: la estandarización de procesos tiene como objetivo fortalecer la habilidad de las mipymes para agregar valor. La estandarización de los procesos mitiga la generación de errores que pueden estar en contra de la inocuidad del producto final y robustecer los procesos productivos y administrativos.

Es necesario conocer los diferentes procesos de la empresa y crear nuevos métodos para mejorar. Debe involucrarse a todo el equipo. Es conveniente utilizar diagramas de flujo, fotografías o dibujos que describan el proceso y generar revisiones, es decir, simplificar la documentación, tratando de mantenerla lo más simple y gráfica posible, difundiendo el uso del proceso estandarizado a todos los colaboradores.

Tecnologías de la información y comunicación: Las TIC, se constituyen como un elemento transversal que permite una manipulación de los datos, información y conocimiento a través de diferentes metodologías que facilitan su captura, registro, conversión, almacenamiento, presentación, administración y transmisión, que se constituyen, técnicamente, como medios para la comunicación entre todos los actores de la cadena del sector lácteo.

Control y seguimiento: el control y el seguimiento es un factor clave para la creación de cultura y generación de compromiso en los colaboradores de las empresas. Este eje crea indicadores y alarmas sobre las desviaciones en las actividades ejecutadas por cada uno de los colaboradores y crea planes de acción oportunos para que las empresas eviten consecuencias como incumplimiento en las entregas y/o entregas con calidad e inocuidad inferior a la esperada.

\section{VALIDACIÓN DEL MODELO PROPUESTO}

Con el fin de extender la propuesta de co-creación de valor en el sector lácteo, se crea la página en Facebook "Innovación para la co-creación en lácteos". El sitio fue desarrollado de acuerdo con la siguiente caracterización:

- Categoría: Servicios de asesoría/negocios

- Nombre: Co-creación para la innovación en lácteos

- Nombre de usuario: www.facebook.com/cocreacionlacteos 
- Sé parte de nuestra base de datos como colaborador y recibe Feedback constantemente que te servirán como herramienta de mejora en tu empresa: http://goo.gl/forms/ ObEQfFU7u9.

- Identificador de página de Facebook: 1714968262113891

Durante el proceso se invitó a empresas del sector, consumidores y demás actores a seguir la página de Facebook donde se publicó información de la industria y de la investigación periódicamente.

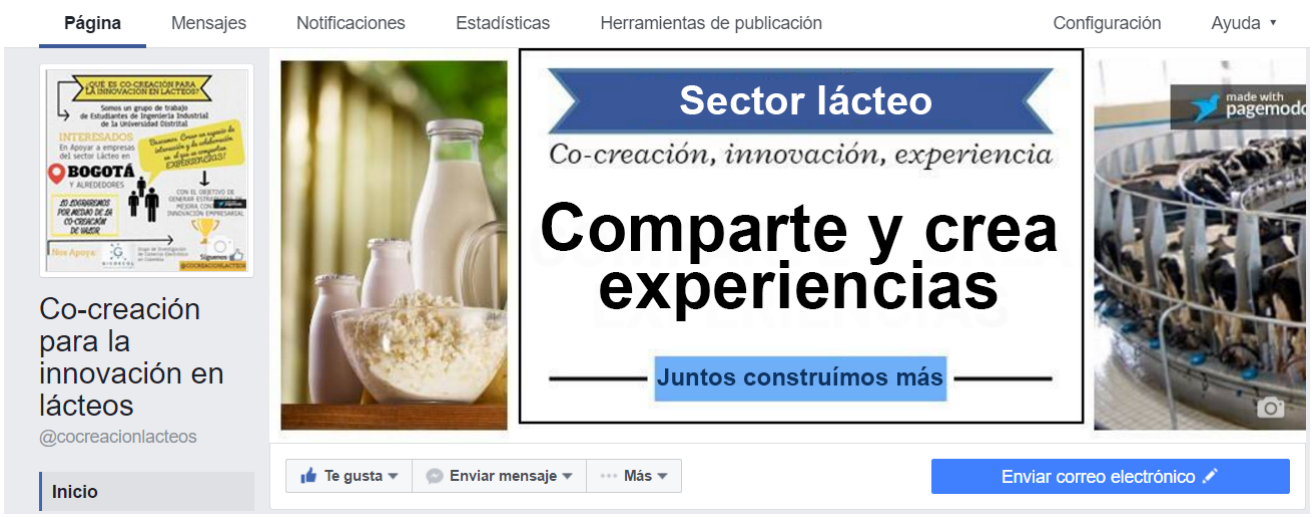

Figura 3. Página Web Co-creación para la innovación en lácteos

Fuente:elaboración propia.

Se realizó un panel con la participación de once expertos. Los expertos fueron citados mediante la participación y cooperación de la Cámara de Comercio de Bogotá -Cluster lácteo- y docentes de la Universidad Distrital Francisco José de Caldas, Facultad de Ingeniería, con el propósito de conocer la opinión de los actores del sector lácteo acerca del modelo propuesto. Para el análisis de la información recopilada se siguió el modelo de análisis planteado por Samaja, Taylor y Bogdan [50].

Los expertos calificaron los criterios generales según la percepción que se tuvo sobre el modelo de co-creación propuesto, se evaluó la presentación, relevancia del contenido, claridad de la información y funcionamiento del modelo.

Se obtuvo un promedio de respuesta positivo del $93,2 \%$, con calificaciones excelente $(61,4 \%$ ) y bueno (31,8 \%); lo anterior indica que la presentación del modelo coherente, mientras que el porcentaje restante no calificó algunos criterios por falta de información u omisión, y por otra parte se recibió una calificación regular en el criterio de presentación visual y gráfica. Hicieron énfasis en que una de las necesidades más críticas del sector lácteo es mitigar la informalidad y el modelo representa muy 
bien la metodología para innovar productos, servicios y procesos pero no representa la reducción de la informalidad.

\subsection{Nivel de aceptación del modelo}

El diseño de las preguntas se realizó como una afirmación que podía ser aceptada o negada por el experto a partir de la información recibida del modelo propuesto. En la siguiente tabla se muestra el porcentaje de respuesta por cada afirmación. La totalidad de los expertos consideran que el modelo podría ser una oportunidad y herramienta de cambio, siempre y cuando se socialice a todos los interesados y se logre generar un clima favorable de sana competencia. Según el panel de expertos, el modelo no fomenta la formalización de las empresas y consecuentemente se afecta la estandarización de procesos y la entrega de productos confiables al cliente mientras se cumple con altos estándares de calidad. Los expertos adicionalmente afirman lo siguiente.

- Las mipymes no obligatoriamente ven la formalidad como una ventaja competitiva.

- Se debe definir los problemas que se incluyen en la informalidad y el plan a seguir para poder solucionar los factores que la propician.

- Es necesario plantear relaciones más evidentes en el modelo y que sean aplicables a las situaciones por las que las empresas se encuentran en la informalidad.

Los aportes realizados por los expertos concluyen que el gobierno es un actor clave y no está participando activamente en las iniciativas que se han adelantado; así el modelo tenga en cuenta a este actor, se debe fortalecer la manera en la que se motiva su participación. Los expertos recomiendan hacer más énfasis en el cambio de cultura para lograr mayor posicionamiento de I+D+i. El $91 \%$ de los expertos afirma que se debe incluir el eje de las tecnologías de la comunicación y la información. Según el concepto de la totalidad de los expertos, el modelo podría llegar a ser implementado a escala local, generando nuevas oportunidades de mercado a través de las estrategias planteadas y motivar el consumo de productos lácteos.

\subsection{Viabilidad de aplicación del modelo}

Para las preguntas generales que hacen referencia a la viabilidad del modelo y a la aplicación de co-creación y gestión del conocimiento a las mipymes del sector, se encontró una respuesta unánime favorable para cada uno de los criterios, concluyendo que el modelo propuesto genera valor, aporta conocimiento y articula la cadena láctea, considerando que es viable su alcance y futura implementación en empresas del sector y es posible su adaptación a otras industrias. Además se confirma que las herramientas de co-creación son aplicables en el entorno empresarial actual del sector. Por parte de 
los empresarios, se sugirió la inclusión de más funciones haciendo énfasis en el aspecto legalidad y tributario, y el establecimiento de un plan pedagógico de implementación en las empresas.

\subsection{Validez de sensibilidad}

Se realizó el contraste entre análisis de sensibilidad realizado en la primera etapa de validación y los resultados obtenidos mediante la aplicación del cuestionario a los expertos en el que se pidió la ponderación del nivel de influencia y relación. Se preguntó: ¿cuál de las relaciones deberían ser el fundamento para el desarrollo del modelo? a partir de las siguientes opciones:

a. Dirección - Estrategia - Planeación de la co-creación

b. Calidad - Autoevaluación -Control y Seguimiento

c. Gestión del conocimiento - Entorno Social - TIC

d. $\mathrm{I}+\mathrm{D}+\mathrm{i}-$ Cultura de Conocimiento - Asociatividad

Se evaluaron las interacciones de los componentes críticos del modelo, el objetivo fue comparar la percepción del modelo por parte de cada experto y profesional desde su perspectiva del sector con el de la investigación.

A nivel global, las respuestas obtenidas indican que la interacción crítica que fundamenta el modelo es $\mathrm{I}+\mathrm{D}+\mathrm{i}$, cultura de conocimiento, asociatividad con un total de selección del $45 \%$, seguida de dirección, Estrategia, y planeación de la co-creación con $36 \%$.

A partir de esta socialización del modelo se realizó una discusión con los expertos en la cual se hicieron diferentes aportes y se identificaron 6 factores fundamentales a tener en cuenta.

1. Es necesario promover una cultura en la que las mipymes deban ser más activas en la búsqueda de información y participación en espacios que les aporten valor. El gobierno y las instituciones del sector deben divulgar y comunicar esta información con el fin de ser incluyentes con los pequeños y medianos empresarios que, así como las grandes empresas aportan al crecimiento del sector. Se debe generar conciencia en los consumidores sobre el paradigma del "precio más bajo" motivando que haya un interés general en el que todos los eslabones conozcan la procedencia y destino de sus productos. 
2. Hay que educar a los consumidores para que reconozcan la calidad de los productos con el objetivo de que se valore la labor de cada eslabón de la cadena y así mismo se traduzca en una retribución económica. El sector está en un cambio generacional en el que es necesario usar la capacitación y educación como herramientas base para formar adecuadamente a los empleados, evitando la rotación de personal y mejorando la calidad de los procesos y productos.

3. La calidad no solo debe reconocerse en el producto final, pues consiste en un proceso continuo que inicia desde el eslabón primario.

La totalidad de los expertos consideran que el modelo podría ser una oportunidad y herramienta de cambio, siempre y cuando se socialice a todos los interesados y se logre generar un clima favorable de sana competencia. Se identificó que la problemática de la informalidad es un factor crítico en el funcionamiento del sector y por tanto toma relevancia en el modelo.

El gobierno es un actor clave que actualmente no está participando activamente en las iniciativas que se han adelantado, así el modelo tenga en cuenta a este actor se debe fortalecer la manera en la que se motiva su participación.

No necesariamente una estructura de costos sostenible es consecuencia de la generación de procesos de $\mathrm{I}+\mathrm{D}+\mathrm{i}$ y cultura de conocimiento, por tanto, es necesario explicar esta relación en el modelo. Como complemento los expertos recomiendan hacer más énfasis en el cambio de cultura para lograr mayor posicionamiento de $\mathrm{I}+\mathrm{D}+\mathrm{i}$. Es importante destacar la observación realizada por el experto en TIC que advierte que debe haber claridad en cómo el modelo a través de las tecnologías de la información generará espacios de co-creación con la cadena del sector.

Según el concepto de la totalidad de los expertos, el modelo podría llegar a ser implementado a escala local generando nuevas oportunidades de mercado y, a través de las estrategias planteadas, motivar el consumo de productos lácteos.

\section{CONCLUSIONES}

El modelo propuesto se considera válido de acuerdo a la aplicación de la metodología propuesta a partir de la evaluación positiva de los expertos y el análisis general de los resultados. Sin embargo, es necesario tener en cuenta factores como la informalidad, que afecta la calidad e inocuidad de los productos y, por ende, la demanda de productos lácteos; afectándose la competitividad de todas las empresas, sean mipymes o grandes compañías. 
La retroalimentación realizada al modelo en las diferentes etapas de validación permite plantear los ajustes pertinentes al modelo, logrando reducir las limitaciones identificadas a partir de su validación. El gobierno y otras instituciones deben estar alineados con una política de legalidad y contribuir a la formación de empresas de acuerdo con las problemáticas que actualmente sufre el sector lácteo.

Como se expuso inicialmente, la innovación y creación de experiencias entre los actores involucrados en el desarrollo del sector lácteo facilita la creación de ventajas competitivas, la integración de los clientes en los procesos de creación o personalización de productos o servicios, incrementa la satisfacción de las necesidades de los clientes, apodera al recurso humano en la innovación, creación de procesos de formación y aprendizaje de los clientes internos y contribución a la mejora de los procesos de las mipymes del sector lácteo; permitiendo superar las barreras existentes para lograr un alto nivel de competitividad.

Se caracterizó el sector lácteo e identificaron factores claves que las empresas utilizan para mejorar continuamente. De igual manera, se identificaron los elementos claves para gestionar la información y conocimiento en dicho sector y se generó una propuesta de modelo para fortalecerlo. Asumiendo que la innovación se ha convertido en el principal agente de crecimiento, un modelo de desarrollo necesariamente tendrá que fortalecer la capacidad para interactuar con todos los actores del sector, producir y aplicar conocimiento en todos los órdenes, teniendo como ejes transversales la planeación, estandarización, las TIC y el control y seguimiento.

\section{REFERENCIAS}

[1] S. Muñoz, "La gestión del conocimiento en las Pymes. Recomendaciones de la Unión Europea," La innovación en México y España, pp. 1-13, 2007.

[2] C. Durugbo and K. Pawar, "A unified model of the co-creation process," Expert Syst. Appl., vol. 41, n. ${ }^{\circ}$ 9, pp. 4373-4387, Jul. 2014.

[3] M. R. S. Maulana and P. Rufaidah, "Co-creation of Small-medium Enterprises," Procedia - Soc. Behav. Sci., vol. 115, pp. 198-206, Feb. 2014.

[4] B. Rodríguez et al., "Propuesta metodológica para analizar la competitividad de redes de valor agroindustriales.," Rev. Mex. Agronegocios, vol. 32, n. 1405-9282, pp. 231-244, 2013.

[5] J. Echeverry, M. Aristizabal, F. Moreno, and A. Bedoya, "Diseño de un sistema difuso para valoración de aportes en sistemas colaborativos," Rev. Ing. Univ. Medellín, vol. 11, n. 1692-3324, pp. 139-152, 2012. 
[6] A. Montoya, I. Montoya, and O. Castellanos, "Situación de la competitividad de las Pyme en Colombia: elementos actuales y retos Current competitiveness of Colombian SMEs: determining factors and future challenges," Agron. Colomb., vol. 28, n. ${ }^{\circ}$ 1, pp. 107-117, 2010.

[7] A. Samson, "Co-creation : New pathways to value An overview," LSE Enterp., vol. 1, pp. $1-21,2009$.

[8] X. Zhang, G. Wang, J. Shangguan, and Y. Xiao, "Value Co-creation with Customers and Its Impact on Capabilities," IEEE IEEM, vol. 08, n. ${ }^{\circ}$ 2007, pp. 2087-2091, 2008.

[9] A. Ruiz Moreno, T. Ortega Egea, C. Haro Domínguez, and M. Roldán Bravo, "El proceso de co-creación de valor y su impacto en la estrategia de innovación en empresas de servicios," Intang. Cap. Univ. Politècnica Catalunya, vol. 10, n. ${ }^{\circ}$ 2014-3214, pp. 266-293, 2014.

[10] M. E. Diz Comesaña and N. Rodríguez López, "Marketing la participación del cliente como co-creador de valor en la prestación del servicio Nuria Rodríguez-López," Innovar. Rev. Ciencias Adm. y Soc., vol. 21, n. ${ }^{\circ}$ 0121-5051, pp. 159-168, 2011.

[11] M. Lehrer, A. Ordanini, R. DeFillippi, and M. Miozzo, "Challenging the orthodoxy of value co-creation theory: A contingent view of co-production in design-intensive business services," Eur. Manag. J., vol. 30, n. ${ }^{\circ}$, pp. 499-509, Dec. 2012.

[12] P. Lugosi, "Mobilising identity and culture in experience co-creation and venue operation," Tour. Manag., vol. 40, pp. 165-179, Feb. 2014.

[13] S. Chakraborty, S. Bhattacharya, and D. D. Dobrzykowski, "Impact of Supply Chain Collaboration on Value Co-creation and Firm Performance: A Healthcare Service Sector Perspective," Procedia Econ. Financ., vol. 11, n. ${ }^{\circ}$ 14, pp. 676-694, 2014.

[14] H. T. Debas and T. J. Coates, "The University of California Global Health Institute opportunities and challenges.," Infect. Dis. Clin. North Am., vol. 25, n. ${ }^{\circ}$ 3, pp. 499-509, viI, Sep. 2011.

[15] M. Kangas, "Creative and playful learning: Learning through game co-creation and games in a playful learning environment," Think. Ski. Creat., vol. 5, n. ${ }^{\circ}$ 1, pp. 1-15, Apr. 2010.

[16] J. Gebauer, J. Füller, and R. Pezzei, "The dark and the bright side of co-creation: Triggers of member behavior in online innovation communities," J. Bus. Res., vol. 66, n. ${ }^{\circ}$, pp. 1516-1527, Sep. 2013.

[17] J. C. Healy and P. McDonagh, "Consumer roles in brand culture and value co-creation in virtual communities,” J. Bus. Res., vol. 66, n. ${ }^{\circ}$ 9, pp. 1528-1540, Sep. 2013.

[18] F. G. H. Behncke, J. Eichinger, and U. Lindemann, "Involvement of Procurement in the Product Creation Process: A Systematization Scheme of Measures," Procedia CIRP, vol. 17, pp. 356-361, 2014.

[19] M. C. Esi, "Formulating the Mission of Business Organization by Reference to the Economic Market Requirements," Procedia Econ. Financ., vol. 20, n. 15, pp. 201-207, 2015. 
[20] G. Tarazona, M. Gomez, and M. Carlos, "Buenas prácticas para implementación del comercio electrónico en pymes,” Visión electrónica, vol. 2, pp. 31-45, 2012.

[21] G. Coates, "Agent co-ordination aided distributed computational engineering design," Expert Syst. Appl., vol. 31, n. ${ }^{\circ}$, pp. 776-786, Nov. 2006.

[22] R. Belderbos, B. Cassiman, D. Faems, B. Leten, and B. Van Looy, "Co-ownership of intellectual property: Exploring the value-appropriation and value-creation implications of co-patenting with different partners," Res. Policy, vol. 43, n. ${ }^{\circ}$ 5, pp. 841-852, Jun. 2014.

[23] G. Tarazona Bermúdez, V. H. Medina García, and L. Giraldo, "Modelo de implementación de soluciones de comercio electrónico," Rev. Ing. Univ. Medellín, vol. 12, n. . 23, pp. 131-143, 2013.

[24] J. J. M. Ferreira, C. I. Fernandes, H. Alves, and M. L. Raposo, "Drivers of innovation strategies: Testing the Tidd and Bessant (2009) model," J. Bus. Res., vol. 68, n. ${ }^{\circ}$ 7, pp. 1395-1403, 2015.

[25] S. H. Bae and K. Yoo, "Economic modeling of innovation in the creative industries and its implications," Technol. Forecast. Soc. Change, vol. 96, pp. 101-110, 2015.

[26] S. Roper and N. Hewitt-Dundas, "Path dependency and innovation: Evidence from matched patents and innovation panel data," Druid 2011 Innov. Strateg. Struct., vol. 44, n. ${ }^{\circ}$ 7, pp. 1327-1340, 2011.

[27] R. Mabudafhasi, "The role of knowledge management and information sharing in capacity building for sustainable development - an example from South Africa," Ocean \& Coastal Management. 2002.

[28] P. Fidel, W. Schlesinger, and A. Cervera, "Collaborating to innovate: Effects on customer knowledge management and performance,” J. Bus. Res., vol. 68, n. ${ }^{\circ}$, pp. 1426-1428, 2015.

[29] Y. Liao, M. Lezoche, H. Panetto, N. Boudjlida, and E. R. Loures, "Semantic annotation for knowledge explicitation in a product lifecycle management context: A survey," Comput. Ind., vol. 71, pp. 24-34, 2015.

[30] M. a. Ale, C. M. Toledo, O. Chiotti, and M. R. Galli, "A conceptual model and technological support for organizational knowledge management," Sci. Comput. Program., vol. 95, pp. 73-92, 2014.

[31] D. A. Vanegas, G. M. Tarazona-bermúdez, and L. A. Rodríguez-rojas, "Mejora de la toma de decisiones en ciclo de ventas del subsistema comercial de servicios en una empresa de IT," Rev. Cient., vol. 38, n. ${ }^{\circ}$ 38, 2020.

[32] R. Anaya Hernández, M. C. Tumino, J. F. Niño Manrique, J. Bournissen, and W. H. Arboleda Mazo, "Motivación de estudiantes de ingeniería en informática con énfasis en ingeniería de software: un estudio en universidades latinoamericanas," Rev. Ing. Univ. Medellín, vol. 19, $n^{\circ} .36$, pp. 239-260, 2020. 
[33] A. Aragón Sánchez, G. Sánchez Marín, and A. Mueses Morales, “Gestión del conocimiento y resultados empresariales: efecto mediador de las prácticas de recursos humanos," Rev. Eur. Dir. y Econ. la Empres., 2015.

[34] V. Zwass, "Co-Creation: Toward a Taxonomy and an Integrated Research Perspective," Int. J. Electron. Commer., vol. 15, n. ${ }^{\circ}$ 1, pp. 11-48, Oct. 2010.

[35] J. Czepiel, E. Gutman, M. Solomon, and C. Surprenant, "A role theory perspective on Dydic interactions: The service encounter," J. Mark., vol. 49, pp. 99-111, 1985.

[36] B. Ertimur, "The Role of Perceived Control in Co-Production," Eur. Adv. Consum. Res., vol. 8, pp. 334-335, 2008.

[37] B. Ertimur and A. Venkatesh, "Opportunism in Co-Production: Implications for Value Co-Creation Burcak,” Australas. Mark. J., vol. 18, pp. 256-263, 2009.

[38] J. H. Schumann, N. V Wünderlich, and M. S. Zimmer, "Culture's Impact on Customer Motivation to Engage In Professional Service Enactments," Cult. impact, vol. 64, n. ${ }^{\circ}$ april, pp. 141-165, 2012.

[39] C. Durugbo and K. Pawar, "Visualising the co-creation process," 18th Int. ICE Conf. Eng. Technol. Innov., vol. 18, pp. 1-10, Jun. 2012.

[40] C. K. Prahalad and V. Ramaswamy, "Co-creation experiences: The next practice in value creation," J. Interact. Mark., vol. 18, n. ${ }^{\circ}$ 3, pp. 5-14, 2004.

[41] T. Redlich, P. Krenz, S.-V. Basmer, S. Buxbaum-Conradi, S. Wulf, and J. P. Wulfsberg, “The Impact of Openness on Value Co-creation in Production Networks," Procedia CIRP, vol. 16, pp. 44-49, 2014.

[42] J. Palacios, G. M. Tarazona-Bermudez, and D. Forero, "Las organizaciones frente a las TIC : conceptualización y características - The Front of Organizational ICT: Concept and Features," Rev. Vinculos, vol. 9, n. ${ }^{\circ}$ 2, pp. 221-232, 2012.

[43] W. Mauser et al., "Transdisciplinary global change research: The co-creation of knowledge for sustainability," Curr. Opin. Environ. Sustain., vol. 5, n. ${ }^{\circ}$ 3-4, pp. 420-431, Sep. 2013.

[44] C. Mele, "Conflicts and value co-creation in project networks," Ind. Mark. Manag., vol. 40, n. ${ }^{\circ}$, pp. 1377-1385, Nov. 2011.

[45] S. Nätti, S. Pekkarinen, A. Hartikka, and T. Holappa, "The intermediator role in value cocreation within a triadic business service relationship," Ind. Mark. Manag., n. IMM-07019, pp. 1-8, May 2014.

[46] S. L. Vargo, P. P. Maglio, and M. A. Akaka, "On value and value co-creation: A service systems and service logic perspective,” Eur. Manag. J., vol. 26, n. 3, pp. 145-152, Jun. 2008.

[47] Propais, "El sector lácteo colombiano,” http://propais.org.co/, 2015. 
[48] A. Jaramillo and A. Segura, "Análisis del mercado de la leche y derivados lácteos en colombia (2008 - 2012)," Supt. Ind. y Comer., p. 100, 2013.

[49] Proexport, "Sector Lácteo en Colombia," 2011.

[50] A. Aparicio, D. W. Palacios, A. M. Martínez, I. Ángel, C. Verduzco, and E. Retana, Métodos de Investiación Avanzada. 2006. 